\title{
Rhythmic Episodes of Subthreshold Membrane Potential Oscillations in the Rat Inferior Olive Nuclei In Vivo
}

\author{
Edith Chorev, ${ }^{1}$ Yosef Yarom, ${ }^{1}$ and Ilan Lampl ${ }^{2}$ \\ ${ }^{1}$ Department of Neurobiology and Center for Computational Neuroscience, Hebrew University, Jerusalem 91904, Israel, and ${ }^{2}$ Department of Neurobiology, \\ Weizmann Institute, Rehovot 76100, Israel
}

\begin{abstract}
In vitro studies of inferior olive neurons demonstrate that they are intrinsically active, generating periodic spatiotemporal patterns. These self-generated patterns of activity extend the role of olivary neurons beyond that of a deliverer of teaching or error signals. However, autorhythmicity or patterned activity of complex spikes in the cerebellar cortex was observed in only a few studies. This discrepancy between the self-generated rhythmicity in the inferior olive observed in vitro and the sporadic reports on rhythmicity of complex spikes can be reconciled by recording intracellularly from inferior olive neurons in situ. To this end, we recorded intracellularly from olivary neurons of anesthetized rats. We demonstrate that, in vivo, olivary neurons show both slow and fast rhythmic processes. The slow process $(0.2-2 \mathrm{~Hz})$ is expressed as rhythmic transitions from quiescent periods to periods of fast rhythm, manifested as subthreshold oscillations of 6-12 Hz. Spikes, if they occur, are locked to the depolarized phase of these subthreshold oscillations and, therefore, hold and transfer rhythmic information. The transient nature of these oscillatory epochs accounts for the difficulties to uncover them by prolonged recordings of complex spikes activity in the cerebellar cortex.
\end{abstract}

Key words: inferior olive; cerebellum; complex spikes; in vivo; intracellular; oscillations; rhythmicity

\section{Introduction}

One classic view of cerebellar function is based on the assumption that the inferior olive delivers an error or a teaching signal to the cerebellar cortex (Marr, 1968; Albus, 1971; Ito, 1982; Lou and Bloedel, 1992). This signal is then used to modulate the strengths of cerebellar inputs in a way that either prevents the reoccurrence of error (Andersson and Armstrong, 1986; Lou and Bloedel, 1986, 1992; Kim et al., 1987) or links a response to a conditioning cue (McCormick et al., 1985; Lou and Bloedel, 1988a,b; Steinmetz et al., 1989). This classical view is challenged by the findings of oscillatory activity intrinsic to the olivary nuclei (Llinas et al., 1974; Llinas and Yarom, 1981a,b, 1986; Lampl and Yarom, 1993, 1997). Such rhythmic activity implies that these nuclei are capable of self-generating regenerative spatiotemporal patterns (Devor and Yarom, 2002a; Leznik et al., 2002). The fact that the neurons are electrically coupled (Llinas et al., 1974; Sotelo et al., 1974) suggests that these patterns can be synchronized (Andersson and Armstrong, 1986; Lou and Bloedel, 1986; Sugihara et al., 1995; Lang et al., 1999; Lang, 2001, 2002; Blenkinsop and Lang, 2006) and therefore carry significant temporal information that

Received Nov. 30, 2006; revised March 14, 2007; accepted April 4, 2007.

This work was supported by the Israel Science Foundation (Y.Y. and Grant 1037/03 to I.L.), the USA-Israel Binational Science Foundation (Y.Y.), the Henry S. and Anne Reich Research Fund for Mental Health (I.L.), and the Asher and Jeanette Alhadeff Research Award (I.L.). I.L. is an incumbent of the Carl Frances Korn Career Development Chair in the Life Sciences. We also thank Jonathan Loewenstein, Eyal Cohen, and Iddo Lev for their comments and advice on this manuscript.

Correspondence should be addressed to Edith Chorev, Department of Neurobiology, Hebrew University, Jerusalem 91904, Israel. E-mail: edithc@lobster.Is.huji.ac.il.

D0I:10.1523/JNEUROSCI.5187-06.2007

Copyright $\odot 2007$ Society for Neuroscience $\quad$ 0270-6474/07/275043-10\$15.00/0 enables it to act as a timing device (Welsh and Llinas, 1993; Yarom and Cohen, 2002).

The properties of these oscillations have been studied by several laboratories using in vitro brainstem slice preparations. In these studies, it has been established that the frequency of these oscillations vary between 1 and $10 \mathrm{~Hz}$ with an amplitude ranging from 3 to $30 \mathrm{mV}$. They are independent of chemical synapses and $\mathrm{Na}^{+}$currents, but are abolished by mibefradil, a specific T-type $\mathrm{Ca}^{2+}$-channel blocker (Placantonakis et al., 2000). The incidence of oscillations, apparently, depends on the integrity of the olivary network (Blenkinsop and Lang, 2006; Placantonakis et al., 2006), formed by electrotonically coupled neurons. The frequency of oscillations, however, has been shown to depend on the size of this network (Placantonakis et al., 2006).

The synchronized rhythmic behavior of olivary output is still under debate (for review, see Kitazawa and Wolpert, 2005). Although all in vitro observations unequivocally reveal subthreshold activity, the autorhythmicity of complex spikes (CSs), recorded from Purkinje cells (PCs) has only been observed in a few studies (Sugihara et al., 1995; Lang et al., 1999) (see Fig. 1). This discrepancy between the subthreshold oscillations in the inferior olive observed in vitro and the sporadic reports of rhythmicity in CSs can only be reconciled by recording intracellularly from inferior olive neurons in situ. In this study, in vivo intracellular recordings from olivary neurons show subthreshold oscillations of membrane voltage, which are organized in epochs, lasting from half a second to several seconds. The transient nature of the oscillatory events can account for the discrepancy between the sporadic reports on CS rhythmicity and the frequent observations of subthreshold oscillations in olivary neurons. 


\section{Materials and Methods \\ Surgery}

Sprague Dawley or Wistar rats $(50-150 \mathrm{~g})$ were initially anesthetized using ketamine $(100 \mathrm{mg} / \mathrm{kg})$ and xylazine $(1 \mathrm{mg} / \mathrm{kg})$ diluted in saline $(50 \%)$ and injected intraperitoneally. For the experiments using halothane anesthesia, tracheotomy was performed and a short tube was inserted for artificial respiration using a mixture of halothane and air $(<0.8 \%)$ at a rate of $100-115$ breaths/min. In the experiments using ketamine-xylazine anesthesia, ketamine was injected intramuscularly, every hour after the initial $2 \mathrm{~h}(100 \mathrm{mg} / \mathrm{kg})$, and xylazine was added every $3 \mathrm{~h}$. The animals were then placed in a stereotaxic device securing the horizontal alignment of the skull dorsal surface. Body temperature was controlled using a heating blanket and a feedback circuit (TC-1000; CWE, Ardmore, PA; or a homemade temperature control unit) to maintain rectal temperature at $37^{\circ} \mathrm{C}$. The heart rate $(240-450$ beats $/ \mathrm{min})$, body temperature $\left(36-38^{\circ} \mathrm{C}\right.$ ), end-tidal $\mathrm{CO}_{2}$ level (only in the halothane experiments), and vibrissa and pinch withdrawal movements were used to monitor the stage of anesthesia. We assessed the anesthesia level used in our recordings to be between stages III-2 and III-3 (Friedberg et al., 1999). Lidocaine was injected to the site of surgery and an incision was made in the skin covering the skull from the lamda to the first vertebrate (C1). The muscles covering the cerebellum and brainstem were retracted and the dura covering the dorsal part of the foramen magnum was removed, exposing the dorsal surface of the brainstem. For extracellular recordings of Purkinje cells (PCs), either urethane $(1.5 \mathrm{~g} / \mathrm{kg}$ ) or ketamine-xylazine (see above) anesthesia were used. A craniotomy of 1-2 $\mathrm{mm}$ in diameter was made lateral to the midline of the cerebellum, exposing vermis folia $6 \mathrm{~b}$ or $\mathrm{c}$. All surgical and experimental procedures were performed in accordance with the Weizmann Institute Animal Care and Use Committee.

\section{Electrophysiology}

Sharp intracellular recordings were performed using Axoclamp 2B, or MultiClamp 700B (Molecular Devices, Foster City, CA). Electrodes with final DC resistance of $80-120 \mathrm{M} \Omega$, were pulled on a Sutter puller (model P-97; Sutter Instruments, Novato, CA) and filled with 2 м K-acetate. The electrodes were inserted into the brainstem 1-2 mm lateral to the obex at an angle of $45^{\circ}$. Figure 1 shows the tract of the electrode at the rostralmost coordinates we used, as seen after fixating and slicing of the brain. The electrode was filled with a fluorescent dye that was injected at the depth where activity of olivary neurons was first detected. After penetration, the brain was protected by covering it with warm agar ( $3 \%$ in saline). Inferior olivary neurons were usually encountered at a depth of $2.5-5 \mathrm{~mm}$. The inferior olive was identified by its characteristic typical calcium spikes (see Fig. 3). All signals were filtered at $3 \mathrm{kHz}$ before digitization at $10-40$ $\mathrm{kHz}$ (using PCI-MIO-16X; National Instruments, Austin, TX) and stored on a personal computer for off-line analysis.

\section{ECG filtering}

In most cases, voltage artifacts caused by heart pulsations were small. Nevertheless, special care was taken in removing these artifacts because the frequency of the heart rate is in the same range as the frequency of the subthreshold oscillations. These artifacts were removed by averaging the recorded voltage using the electrocardiogram (ECG) peaks as a triggering signal. We assumed that neuronal activity was uncorrelated to ECG activity and that the ECG artifact was constant. Therefore, the ECG artifact could be subtracted from the voltage segments. For each heartbeat, a corresponding voltage segment was determined. This segment was then averaged over similar 100 preceding and following segments. The average signal was considered to be the heartbeat artifact and was subtracted from the voltage segment. This procedure was repeated for each heartbeat.

\section{Spike sorting}

Single unit recordings were high-pass filtered $(100 \mathrm{~Hz}$ as cutoff frequency) and then sorted (homemade software programmed using Matlab 7.1; MathWorks, Natick, MA) to distinguish simple from complex spikes. Threshold was used to detect spikes. Principal component analysis, together with K-means algorithm, was used to separate the waveforms of simple spikes from the complex spikes (see supplemental Fig. 1, available at www.jneurosci.org as supplemental material). Interspike in-

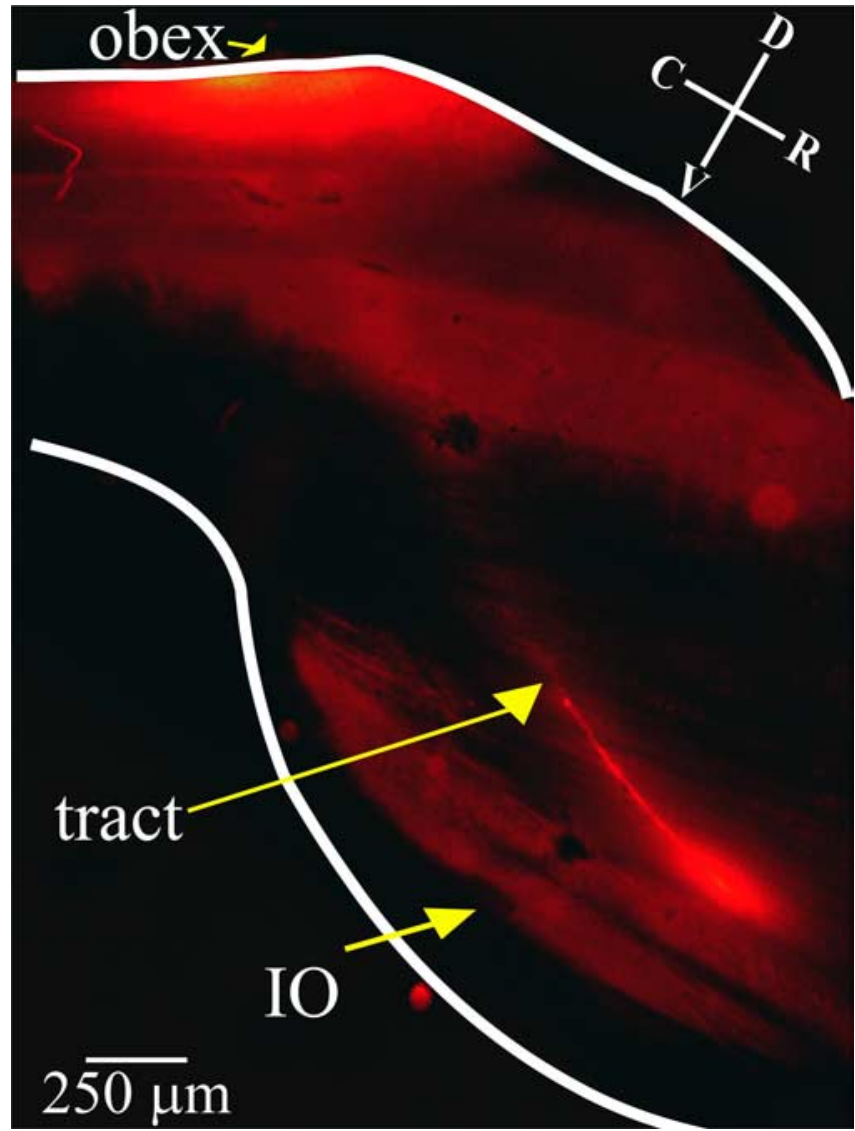

Figure 1. Fluorescent labeling of the electrode tract and recording site. After olivary recording, we inserted an electrode filled with a fluorescent marker (RH414; Invitrogen, Eugene, OR) to the rostralmost coordinates we use for olivary recordings. The animal was then perfused with PBS and then with PFA; $100 \mu \mathrm{m}$ slices were prepared and mounted on microscope slides. The labeling was detected using a fluorescence microscope. The marked areas are the site of penetration (the obex), the tract of the electrode and the injection site, which is at the rostralmost side of the inferior olive. Notice that the entire olive is marked by fluorescence. I0, Inferior olive; $D$, dorsal; V, ventral; C, caudal; $R$, rostral.

terval (ISI) histograms were used to verify refractoriness to assure that it was indeed a single-unit recording. The characteristic complex spike pause was detected by calculating the cross-correlograms between the complex and simple spikes (see supplemental Fig. 1, available at www. jneurosci.org as supplemental material).

\section{Spectral analysis}

The power spectrum and normalized autocorrelations were calculated for all traces. To calculate the ISI, we identified the times of spike occurrence using threshold; the time of spike was defined as the deflection point in the upstroke of the spike. The times of spike occurrence were also used to calculate spike autocorrelations (after binning the data to bin sizes corresponding to the minimal spike duration, which was $\sim 8 \mathrm{~ms}$, and generating a binary vector). All data analysis was performed using Matlab7.1 (MathWorks). All average values are given with the SD (mean $\pm \mathrm{SD}$ ).

Detection of oscillatory epochs. Spikes were clipped from the voltage traces by substituting the spike with a constant voltage. Spikes were removed from the point of initiation (the deflection point) to the point were the voltage crossed this same value on the falling phase of the spike. The signal was then low-pass filtered with $50 \mathrm{~Hz}$ as the cutoff frequency. A window of 1-2 s was used for spectral analysis. For each window, the power spectrum was calculated. If a significant peak was located between 6 and $14 \mathrm{~Hz}$ (peak larger than 2 SDs of the baseline that was calculated from the mean between 14 and $25 \mathrm{~Hz}$ ), it was selected for additional analysis. Consecutive significant segments were merged and a shorter 

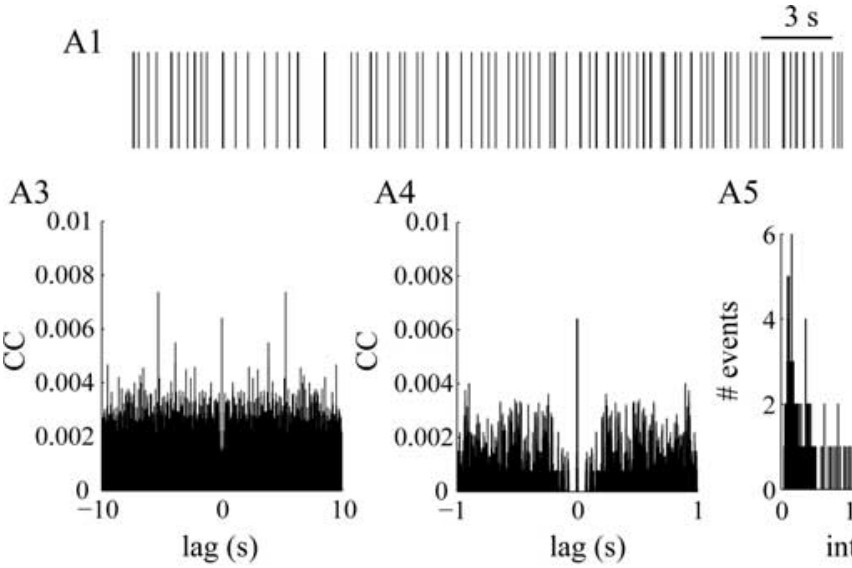

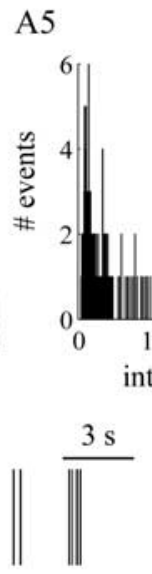

B5
$\mathrm{A} 2$

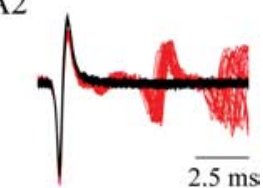

B1

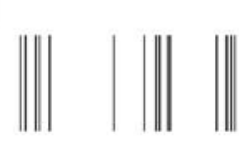

B3

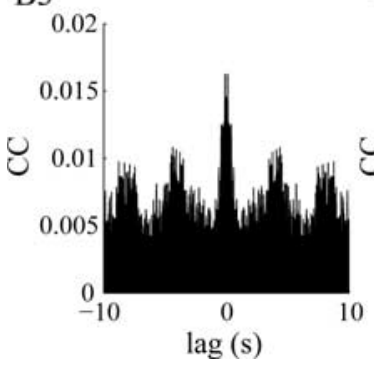

B4
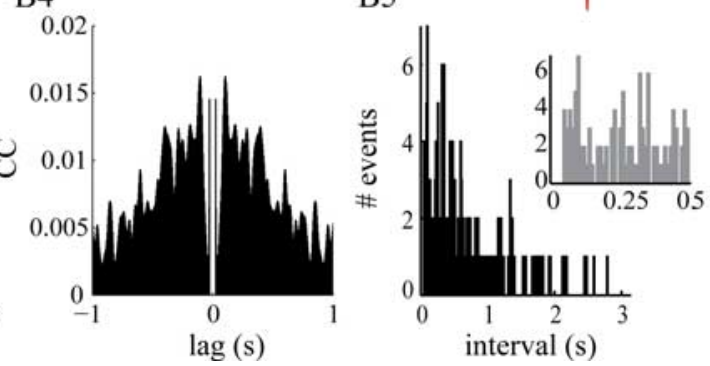

Figure 2. The rarity of $10 \mathrm{~Hz}$ rhythmicity in $\mathrm{CS}$ activity recorded from Purkinje cells. $\boldsymbol{A}, \boldsymbol{B}$, Two examples of $C S$ activity recorded from Purkinje cells. Each example contains the raster plot (1), the CS and simple spikes wave forms (red and black traces; 2), autocorrelograms of the CS at slow (3) and fast (4) time scales, and the histogram of the ISI (5). Note that in both examples, a peak in the ISI histogram is evident (110 and $120 \mathrm{~ms}$ in $\boldsymbol{A}$ and $\boldsymbol{B}$, respectively), but only in $\boldsymbol{B}$ a corresponding fast rhythm was detected in the autocorrelogram. A slow rhythm is also apparent only in $\boldsymbol{B}$. CC, Correlation coefficient.

window of analysis was implemented. Repeating this procedure with shorter windows (down to $200 \mathrm{~ms}$ ) enabled us to determine the beginnings and ends of the oscillatory epochs and to calculate their frequencies and durations.

Calculation of rhythmicity index. The autocorrelation of the binary vector of spike occurrence (see above) was calculated and smoothed with a running average filter of 3 bins (each bin is of $8-10 \mathrm{~ms}$ depending on the duration of the spike) for the faster- and slower-frequency rhythmicity indices (RIs). The first lateral peak for the slow and fast rhythms was determined $\left(t_{1}\right.$ and $t_{2}$, respectively). A Pwelch analysis was performed on the autocorrelogram (window, $10 \mathrm{~s}$ ) to calculate the power spectrum. For the slow rhythm, the power spectrum was normalized to have an integral of 1 . The $\mathrm{RI}_{\text {slow }}$ is the integral calculated at a range of $\pm 0.1 \mathrm{~Hz}$ around the determined frequency $\left(f_{1}=1 / t_{1}\right)$. For the fast rhythms, the integral of the power spectrum between 3 and $50 \mathrm{~Hz}$ was normalized to 1 . $\mathrm{RI}_{\text {fast }}$ was calculated in a similar manner at a range of $\pm 0.5 \mathrm{~Hz}$ from the determined frequency $\left(f_{2}=1 / t_{2}\right)$. Thus, the range of RI was between 0 and 1 .

A similar procedure was used to calculate the subthreshold rhythm. The autocorrelation was calculated for the voltage trace as opposed to spike times. Spikes were clipped (see above) from the voltage traces that were low-pass filtered with $30 \mathrm{~Hz}$ as the cutoff frequency.

\section{Results}

As stated above, the rhythmic appearance of complex spikes has sparked much debate. The data shown in Figure 2 demonstrate that in most of the PCs the CS activity was arrhythmic. Figure 2 A1 shows a raster plot of spontaneous complex spike activity recorded in vivo from a PC. CSs were sorted by their waveforms shown in Figure $2 A 2$ (red traces). The autocorrelogram of this activity (Fig. 2A3) failed to reveal any sign of rhythmicity either at the slow (Fig. 2A3) or fast (Fig. $2 A 4)$ time scales. However, the ISI (Fig. 2A5) demonstrated a clear peak at $110 \mathrm{~ms}$. Occasionally ( 4 of 27 cells, the criterion for rhythmicity was $\mathrm{RI}_{\text {slow }} \geq 0.3$ ), the complex spike activity displayed a slow rhythm $(0.24 \mathrm{~Hz})$ (Fig. $2 \mathrm{~B})$. In this example, the 10 $\mathrm{Hz}$ rhythmicity is also apparent both in the autocorrelogram (Fig. 2 B4) and in the ISI (B5). These observations reflect the controversy regarding the rhythmicity of cerebellar complex spike activity: in most of the recordings, the ISI showed a clear peak at $\sim 100 \mathrm{~ms}$, whereas the autocorrelograms failed to demonstrate $10 \mathrm{~Hz}$ rhythmicity. Similar results have been reported by many laboratories on a variety of preparations after different anesthetic procedures (for review, see Kitazawa and Wolpert, 2005). In an attempt to resolve this controversy, we measured, in vivo, the activity of olivary neurons intracellularly.

Of a total of 50 olivary neurons that were recorded during this study, we selected 32 cells for additional analysis. These cells had a resting potential lower than $-45 \mathrm{mV}$ and an over shooting action potential, and the recording lasted $>2$ $\mathrm{min}$; the longest record lasted for $45 \mathrm{~min}$. Twenty-two cells were obtained under halothane anesthesia and exhibited oscillatory properties, which are described below. Because high concentrations of halothane were reported to block gapjunctional communication in cultures (Wentlandt et al., 2005, 2006), we also reordered from 10 cells under ketamine-xylazine anesthesia. The two populations did not differ in their electrical behavior and spectral profile and, therefore, the data were pooled. Table 1 gives the population properties and a summary for all of the different experiments performed in this study. The average resting potential was $-62 \pm$ $10 \mathrm{mV}(n=32)$ and the input resistance was $42 \pm 8 \mathrm{M} \Omega(n=5)$ (Table 1 ). As with the in vitro preparation, olivary neurons are characterized by two distinct regenerative responses: one has a high-threshold and prolonged action potential (Fig. $3 A$ ) that is likely to be generated at the dendritic level; the other regenerative response has a low-threshold, short-duration action potential that is generated at the somatic level from a more negative membrane potential (Fig. 3C) (Llinas and Yarom, 1981b). The highthreshold spikes reached an average amplitude of $69 \pm 8 \mathrm{mV}(n=$ $32)$ and a half-amplitude duration of $3.7 \pm 4 \mathrm{~ms}(n=32)$ (Table 1). The latter is shorter than that observed in in vitro preparations (Llinas and Yarom, 1981a), as expected from the lower input resistance encountered in vivo. The plateau-like phase of the high-threshold spikes was usually crowned by $2-5$ fast positive wavelets (Fig. 3A, arrow heads). It is likely that these are antidromic invasions of axonal spikes (Crill and Kennedy, 1967; Crill, 1970). Spontaneous low-threshold spikes were rarely observed. They were readily evoked by intracellular current injections 
provided that the membrane was hyperpolarized to levels below $-78 \mathrm{mV}$. On these occasions they had an average amplitude of $67 \pm 4 \mathrm{mV}$ and a duration at half amplitude of $0.5 \pm 0.08 \mathrm{~ms}(n=5)$ (Table $1)$. The prevalence of high-threshold action potentials can be attributed to the in vivo conditions where the dendrites are more depolarized than in the in vitro conditions. It is also likely that in the in vivo conditions, low-threshold $\mathrm{Ca}^{2+}$ current can directly activate the high-threshold action potential. The significant prepotentials (Fig. 3B, arrow) observed in some of the high-threshold action potentials support this suggestion.

The rate of the spontaneous action potentials was $2.08 \pm 1.64 \mathrm{~Hz}(n=32)(\mathrm{Ta}-$ ble 1 ), within the reported range of CS firing frequency recorded extracellularly from PCs $(1.52 \pm 0.89 \mathrm{~Hz})$ (Lang et al., 1999) (Fig. 2). Despite the low rate of spontaneous activity, the population ISI histogram obtained from 32 cells displayed a clear peak at $95 \mathrm{~ms}$ (Fig. 3D, inset). Prolonged recordings revealed that the spikes were not uniformly distributed, but rather were clustered in distinct groups separated by quiescent periods. Within clusters, the typical ISI was $\sim 95 \mathrm{~ms}$ (average peak of ISIs, $95 \pm 45 \mathrm{~ms} ; n=32$ ) (Table 1). This characteristic clustered firing accounts for the discrepancy between the low average firing $(2.08 \mathrm{~Hz})$ rate and the rather short typical ISI (95 ms).

Three examples of prolonged recordings are shown in Figure 4. In some neurons, the spike clusters appear rhythmical (Fig. $4 A$, top trace) and the spectral analysis of the membrane potential revealed a prominent component at $0.2-2 \mathrm{~Hz}$ (Fig. $4 B$, top). In other examples where the firing activity seems to be disorganized (Fig. $4 A$, bottom two traces), the spectral analysis still revealed the underlying slow rhythm (Fig. $4 B$, bottom). In 16 neurons, the spikes occurred during waves of depolarization (Fig. $4 A$, top and middle traces). The other 16 neurons showed spiking activity during periods of large membrane-potential fluctuations, but without any apparent depolarization (Fig. $4 A$, bottom trace). In both cases, the spectral analysis (Fig. $4 B$ ) of the membrane potential shows a significant component at the $5-13 \mathrm{~Hz}$ range. Hence, the spectral content has a characteristic profile: a large component between $0.2-2 \mathrm{~Hz}$ (average frequency $0.83 \pm 1.55 \mathrm{~Hz}$; $n=32$ ) (Table 1 ) and an additional component at the $5-13 \mathrm{~Hz}$ range (average frequency $8.3 \pm 2.4 \mathrm{~Hz} ; n=32$ ) (Table 1). The low frequencies seem to correspond to the appearance of spike clusters, whereas the higher frequencies might correspond to the rhythmicity within a cluster.

It is noteworthy that, in addition to the spikes and the rhythmic changes in membrane potential, we also identified typical spikelet-like signals (Fig. $4 A$, insets). These brief (2-10 ms) de-

B the histogram).
Table 1. Summary of properties and experimental results

\begin{tabular}{|c|c|c|}
\hline Analysis & $n$ & Value \\
\hline Resting potential & 32 & $-62 \pm 10 \mathrm{mV}$ \\
\hline$R_{\text {in }}^{a}$ & 5 & $42 \pm 8 \mathrm{M} \Omega$ \\
\hline High-threshold $\mathrm{Ca}^{2+}$ spike amplitude & 32 & $69 \pm 8 \mathrm{mV}$ \\
\hline High-threshold $\mathrm{Ca}^{2+}$ spike duration & 32 & $3.7 \pm 4 \mathrm{~ms}$ \\
\hline 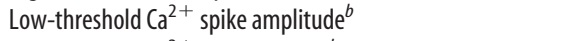 & 5 & $67 \pm 4 \mathrm{mV}$ \\
\hline Low-threshold $\mathrm{Ca}^{2+}$ spike duration $^{b}$ & 5 & $0.5 \pm 0.08 \mathrm{~ms}$ \\
\hline Spontaneous firing rate & 32 & $2.08 \pm 1.64 \mathrm{~Hz}$ \\
\hline Peak of population ISI & 32 & $95 \pm 45 \mathrm{~ms}$ \\
\hline Mean slow frequency (subthreshold) & 32 & $0.83 \pm 1.55 \mathrm{~Hz}$ \\
\hline Mean high frequency (subthreshold) & 32 & $8.3 \pm 2.4 \mathrm{~Hz}$ \\
\hline Percentage of cells with slow rhythmicity (suprathreshold) ${ }^{c}$ & 27 & $63 \%$ \\
\hline Mean slow frequency (suprathreshold) & 17 & $0.65 \pm 0.36 \mathrm{~Hz}$ \\
\hline Percentage of cells with fast rhythmicity (suprathreshold) ${ }^{c}$ & 27 & $30 \%$ \\
\hline Mean fast frequency (suprathreshold) & 9 & $9 \pm 1.7 \mathrm{~Hz}$ \\
\hline Mean oscillations frequency during epochs & 32 & $10.14 \pm 2.00 \mathrm{~Hz}$ \\
\hline Mean oscillatory epoch duration & 32 & $0.9 \pm 0.14 \mathrm{~s}$ \\
\hline Percentage of IPIs that differ in $<0.2$ cycles from the typical IPI & 32 & $41 \pm 14 \%$ \\
\hline
\end{tabular}

${ }^{a}$ Systematic $I-V$ curves were performed only on five cells and only those were used for measuring $R_{\text {in }}$

In the suprathreshold rhythmicity analysis, only cells with a firing rate $>0.3 \mathrm{~Hz}$ were included. 0 f these, 17 cells showed the slow rhythm and nine showed
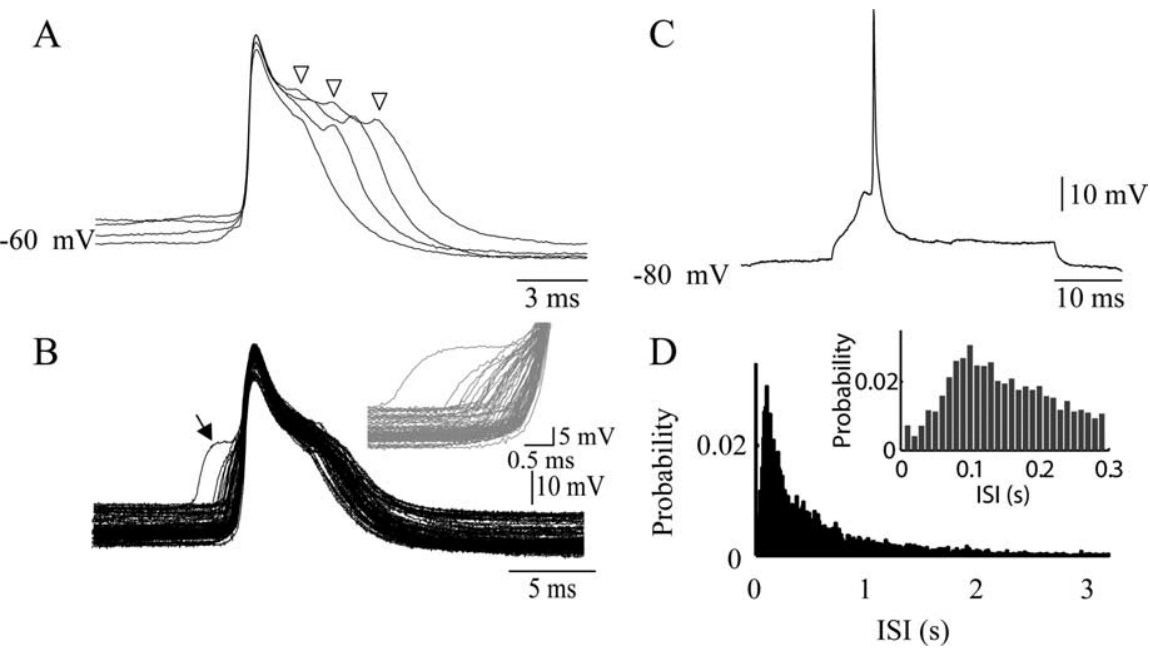

Figure 3. Two types of action potentials recorded from olivary neurons in vivo. $\boldsymbol{A}$, Four superimposed high-threshold calcium spikes of various durations recorded from the inferior olivary neuron. Arrowheads denote wavelets that are likely to represent phase of all action potentials is shown in the inset. The arrow indicates the prepotentials that are presumably a low-threshold calcium component of the action potentials. Note that all of the action potentials are of the high-threshold type. C, Low-threshold average ISI histogram from 32 neurons showing a peak at $95 \mathrm{~ms}$ (inset shows an enlargement of the area surrounding the peak of

polarizing signals appear mostly during the oscillatory epochs of membrane potential. The average frequency of these spikelet-like signals was $1.8 \mathrm{~Hz}$ and it decreased by $10-30 \%$ with $20 \mathrm{mV}$ hyperpolarization. Their amplitude ranged from 1 to $15 \mathrm{mV}$. The low amplitude and fast rise time, together with the dependence on membrane potential, suggest that these events are correlates of intrinsic regenerative responses. A more detailed study is needed to explore the origin of these signals and to understand their functional significance.

The rhythmicity of olivary firing was estimated from the autocorrelation function (see Materials and Methods). This analysis (limited to cells with a firing rate of $0.3 \mathrm{~Hz}$ and higher) revealed different degrees of rhythmicity and supports the assumption that two rhythmic processes govern olivary activity. The exam- 
A
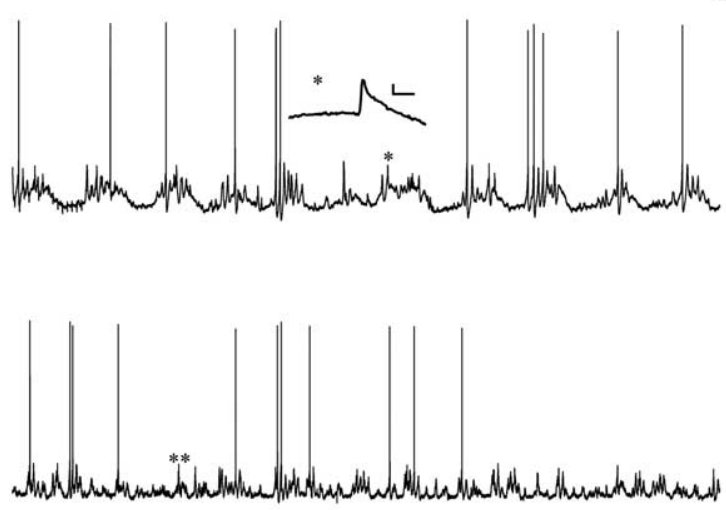
$\overbrace{}^{* *}$

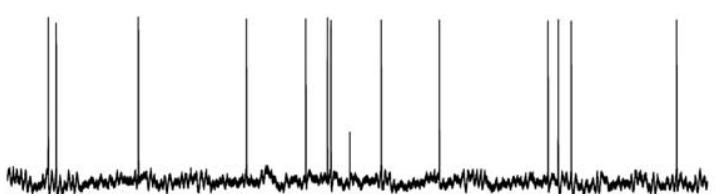

B
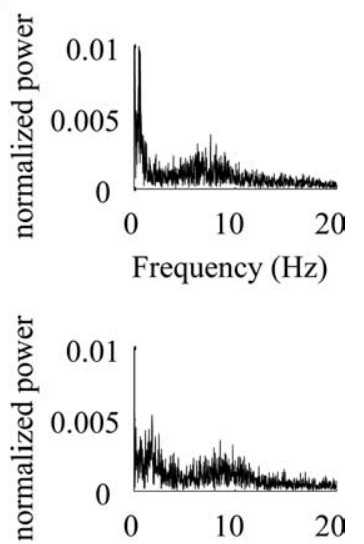

Frequency $(\mathrm{Hz})$

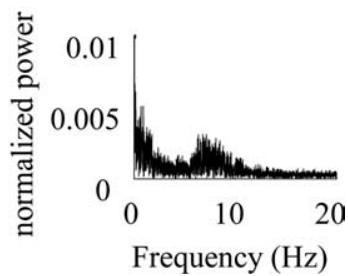

Figure 4. Spectral analysis of olivary neurons reveals two regions of increased power. $A$, Three examples of 20 s recordings from olivary neurons at their resting potential $(-68,-60,-63 \mathrm{mV}$, top to bottom, respectively). $\boldsymbol{B}$, The corresponding power spectra of the three examples shown in $\boldsymbol{A}$. Two regions of increased power are seen in all three cases; the first region is between $0.2-2 \mathrm{~Hz}$ and the second between 5 and $13 \mathrm{~Hz}$. Insets in $\boldsymbol{A}$ show an enlarged display of spikelet-like events (asterisks). Calibration: $2 \mathrm{mV}, 5$ ms. Note that the sharp peak of the slow rhythm in the first example is at $0.5 \mathrm{~Hz}$. In this case, the animal was respirated at $1.6 \mathrm{~Hz}$.

ples depicted in Figure 5 demonstrate the variability in the rhythmicity of firing. The autocorrelograms (Fig. $5 B$ ) reveal different degrees of rhythmicity within the range of the slow frequencies found in the spectral analysis (Fig. 4). The degree of rhythmicity was estimated by calculating the RI (see Materials and Methods). For example, whereas the top autocorrelogram has an $\mathrm{RI}_{\text {slow }}$ of 0.5 , the autocorrelogram at the bottom shows an $\mathrm{RI}_{\text {slow }}$ of 0.01 . The frequency of this slow rhythm ranged between 0.2 and $1.8 \mathrm{~Hz}$ $(0.65 \pm 0.36 \mathrm{~Hz} ; n=27)$ (Table 1), corresponding to the slow frequency in the spectral analysis (Fig. 4). Expanding the central peak of the autocorrelogram (Fig. 5 C) occasionally (in 30\% of cells; $n=27$ ) revealed a fast rhythm that corresponds to the high-frequency range of the spectrum. This high-frequency range of rhythmicity was also manifested in the corresponding ISI (Fig. $5 D$ ). A clear peak at $\sim 100 \mathrm{~ms}$ is evident ( $95 \pm 45 \mathrm{~ms} ; n=32$ ) (Fig. $3 D$ ) in all of the examples, regardless of the degree of rhythmicity. A second peak at rather long intervals is found only in those cases ( $n=3$ of 27) where relatively strong rhythmicity was found and the period of the rhythmic process was relatively short (Fig. 5, middle).

A close examination of the spiking episodes (Fig. 6) revealed that these spikes were elicited from depolarized waves organized in oscillatory epochs (Fig. 6B). Although all of the spikes occurred during the peaks of the depolarized phases (Fig. 6C), many of the oscillatory waves did not elicit spikes. It should be noted that two spikes might occur on a single oscillatory phase, resulting with a short $(<30 \mathrm{~ms})$ ISIs. Such events were observed in 50\% of the cells and in those cells, $3.3 \pm 2.8 \%(n=16)$ of the ISIs corresponded to frequencies of $30 \mathrm{~Hz}$ or higher.

To quantify the frequency, durations and rhythmicity of the oscillatory epochs, we calculated the autocorrelation of the oscil- latory periods and averaged them over the entire recording time (one such example is shown in Fig. 6D). The distribution of the epoch frequencies (measured from the power spectrum calculated for each epoch) for the same cell as in Figure $6 \mathrm{~A}$ is shown in Figure $7 A$ (top). The average frequency of the oscillatory epochs in this example was $10.5 \mathrm{~Hz}$ and the range was 9-12 $\mathrm{Hz}$ (Fig. $7 A$, top). The frequency range over the entire population was $6-12 \mathrm{~Hz}$ (Fig. $7 B$ ). Interestingly, the range of frequencies within any given cell was smaller than the range found in the population, supporting a role for the size of the coupled olivary network in affecting the frequency (Placantonakis et al., 2006). As shown in Figure $7 C$, the duration of these oscillatory epochs (see Materials and Methods) varied from 0.2 to $3 \mathrm{~s}$, displaying a normal distribution (using Kolmogorov-Smirnov test, $\alpha=0.05, p<0.05)$. The average epoch duration calculated from 32 cells was $1.12 \mathrm{~s}$. It should be noted that, unlike the distribution of the frequencies, which was smaller in individual cells than in the entire population, the distribution of the duration of oscillatory epochs in individual cells was similar to that calculated for the entire population.

The fast rhythmic component during the depolarized epochs is seen both in the suprathreshold and subthreshold levels (Fig. 6E,F). The spikes appear to be riding on top of subthreshold oscillations (Fig. $6 B, C$ ). To test whether changing the resting potential below spike threshold diminishes or alters the frequency of the subthreshold rhythmicity, we hyperpolarized the membrane potential of the neurons. One example of such an experiment is shown in Figure 8. Although hyperpolarizing the membrane potential (from -68 $\mathrm{mV}$ at rest to $-87 \mathrm{mV}$ ) prevented firing (Fig. 8, compare $A, B$ ), both the slow and fast rhythms were not affected. The voltage insensitivity of the rhythmic processes is further emphasized in the calculated autocorrelograms shown in Figure $8 C$. The slow frequency was $0.45 \mathrm{~Hz}$ and the $\mathrm{RI}_{\text {slow }}$ was 0.24 . After hyperpolarizing the membrane, the frequency of the rhythm was $0.52 \mathrm{~Hz}$ and the $\mathrm{RI}_{\text {slow }}$ was 0.26 . Similarly, the fast rhythm was also insensitive to the membrane potential changes as demonstrated in Figure $8 D\left(7.41 \mathrm{~Hz}\right.$ and $\mathrm{RI}_{\text {fast }}=0.66$ in rest; $7.55 \mathrm{~Hz}$ and $\mathrm{RI}_{\text {fast }}=$ 0.72 when hyperpolarized). None of the five cells examined at hyperpolarized levels (at least $10 \mathrm{mV}$ hyperpolarization) showed a significant change in either the rhythmicity index or the frequencies of the rhythms $(\alpha=0.005, p>0.8$, paired $t$ test; average change in $\mathrm{RI}_{\text {slow }}$ and $\mathrm{RI}_{\text {fast }}$ was $0.005 \pm 0.024$ and $0.16 \pm 0.12$, respectively; changes in slow and fast frequencies were $0.04 \pm$ 0.06 and $0.03 \pm 0.06 \mathrm{~Hz}$ respectively). Thus, we can conclude that the subthreshold activity is independent of the suprathreshold activity and that the spikes are triggered by the subthreshold oscillations.

The oscillations observed in olivary neurons in vitro were reported to be very precise and, thus, the phase of each cycle can be well predicted (Devor and Yarom, 2002b). To test whether these characteristics are also extended to the in vivo conditions, we examined the precision of subthreshold activity, their modula- 
A

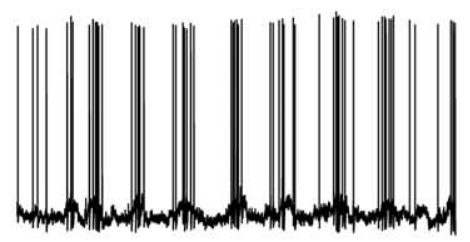

B

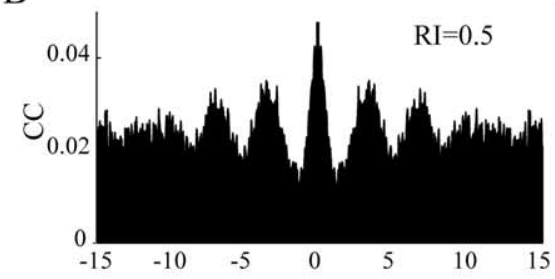

$\mathrm{C}$

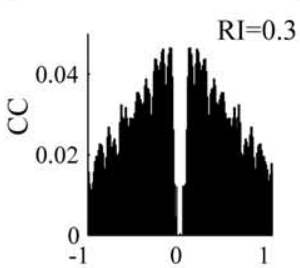

$\mathrm{D}$

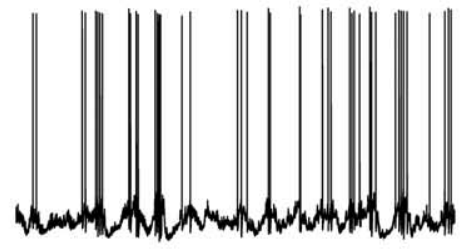

$20 \mathrm{mV} \frac{\mathrm{L}}{2 \mathrm{~s}}$

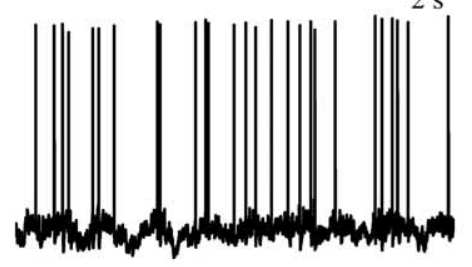

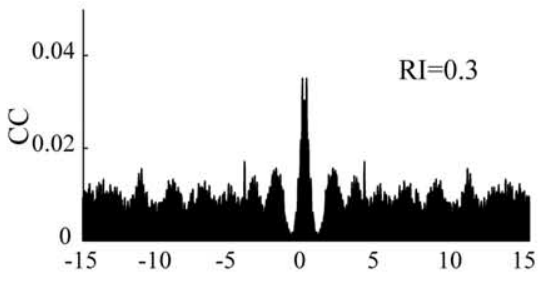
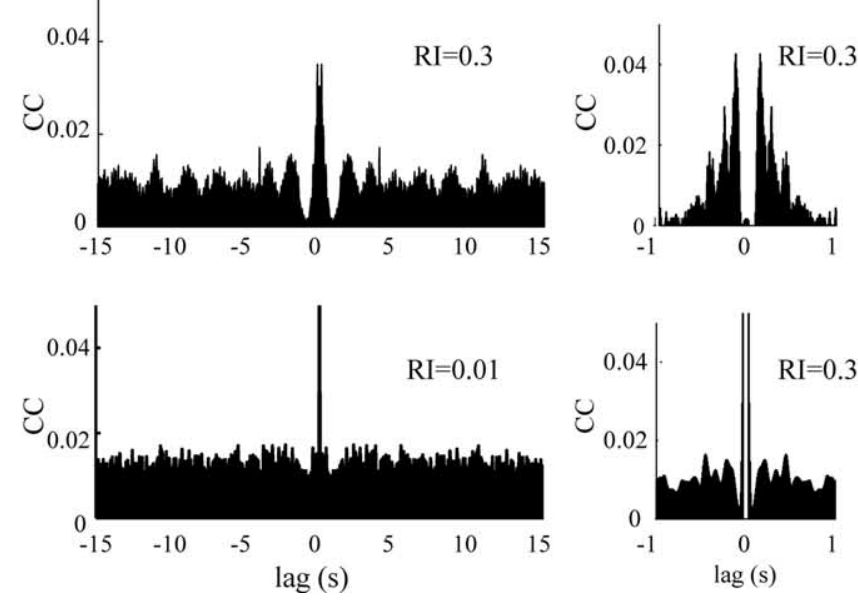



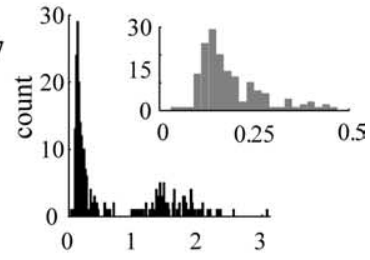
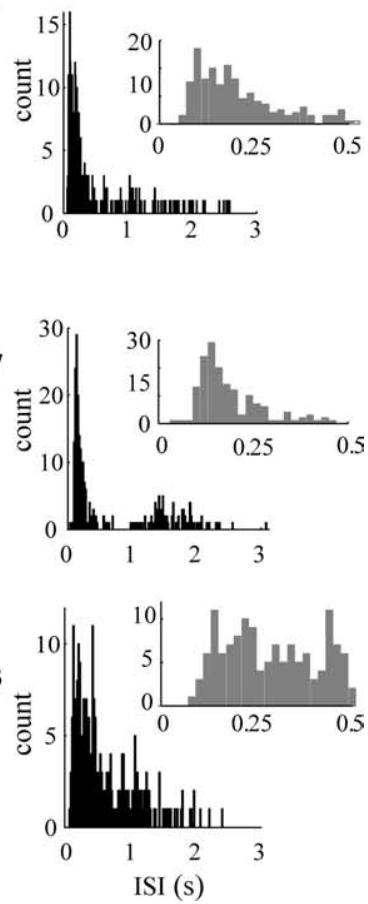

Figure 5. Suprathreshold activity shows two rhythmic processes. $\boldsymbol{A}$, Twenty second recordings from three olivary neurons recorded in absence of injected current. $\boldsymbol{B}$, The corresponding spike autocorrelograms. $\boldsymbol{C}$, The corresponding autocorrelograms on a faster time scale. $\boldsymbol{D}$, The corresponding ISI histograms at low and high (insets) resolutions. Note the slow rhythm in the top two examples in $\boldsymbol{B}$ ( 0.3 and $0.52 \mathrm{~Hz}$; top and middle, respectively) quantified by the RI. A faster rhythm is evident in $\boldsymbol{C}$, which is correlated with the peaks of the ISI histograms ( $\boldsymbol{D}$ ). Firing rates of the cells shown are: $0.48 \pm 0.56,0.864 \pm 1.13$, and $1.53 \pm 1.99 \mathrm{~Hz}$, top to bottom, respectively. CC, Correlation coefficient.

tion by action potentials, and their relationships with suprathreshold activity. To this end, we measured the interpeak intervals (IPIs) of the subthreshold activity throughout the recordings and normalized for each cell by its most frequent interval. The resulting distributions from four cells are shown in Figure 9. The sharp peaks in the distributions of the IPIs (IPIs that do not follow spike, empty bars), suggests a narrow distribution. This sharp peak is reflected in the cumulative distribution histogram by the steep rise of the curve (black curves in insets). The steepness of the curves indicates that most of the IPIs have a similar duration. The percentage of IPIs that differed from the characteristic IPI in $<0.2$ cycles, was $41 \pm 14 \%(n=32)$ (Table 1$)$. The distribution of IPI durations also shows that longer durations of IPIs are not necessarily multiplications of the characteristic interval, suggesting that the oscillatory epochs are independent.

We reason that the subthreshold oscillations are a network phenomenon and, thus, the IPIs should not be affected by a preceding action potential. We therefore measured the distribution of IPIs that followed an action potential, measured from the onset of the spike to the following peak (Fig. 9, yellow bars). The resultant distributions are very similar to the distributions of all IPIs (compare orange and empty bars), suggesting first that spikes are locked to the fast oscillatory activity and, second, that spontaneous action potentials, when they occur, do not affect the underlying subthreshold rhythmicity. As a matter of fact, in most cases, the cumulative distributions of cycles after spikes are steeper than for all cycle periods, as indicated in the cumulative distributions in Figure 9, $A-C$. The percentage of IPIs that follow a spike that differed from the characteristic IPI in $<0.2$ cycles, was $45 \pm 17 \%$ $(n=32)$ (Table 1$)$, similar to the value for all IPIs. If spikes coincide with the oscillatory wave, we expect an overlap between the distributions of the ISIs (blue bars) and the IPIs (empty bars).
Indeed, we found that the distributions overlap with the ISIs tending to have lower probabilities than the IPIs, reflecting the low firing rate. These findings, together with the voltage independence of the frequency of oscillations, support the argument for the network origin of subthreshold oscillations.

\section{Discussion}

In this study, we demonstrate that olivary neurons in vivo display a slow and a fast rhythmic process. The first has a prolonged time scale, manifested as rhythmic transitions $(0.2-2 \mathrm{~Hz})$ from quiescent periods to periods of subthreshold oscillations. This slow rhythm is expressed both in the subthreshold and firing activities. The fast rhythm is manifested as epochs of 6-12 Hz subthreshold oscillations. Spikes, when they occur, are locked to the depolarized phase of these subthreshold oscillations. Hence, action potentials tend to occur with an interspike interval of $95 \mathrm{~ms}$ (Fig. 3D).

The network origin of the oscillatory activity has been suggested previously and several models were put forward to explain how this can be achieved (Manor et al., 1997; Makarenko and Llinas, 1998; Schweighofer et al., 1999; Lowenstein et al., 2005). Of the different models, the nonhomogenous population model (Manor et al., 1997) has been experimentally supported previously (Chorev et al., 2006). This model demonstrated how a heterogeneous population of quiescent neurons can generate network oscillations after being electrotonically coupled. Previous works have shown that disrupting the integrity of the olivary network abolished oscillations or CS rhythmicity (Blenkinsop and Lang, 2006; Placantonakis et al., 2006). In the current study, we demonstrate that hyperpolarizing the membrane potential did not alter the rhythmic processes in olivary neurons (Fig. 8). Thus, the intrinsic properties of the manipulated cells are un- 
A
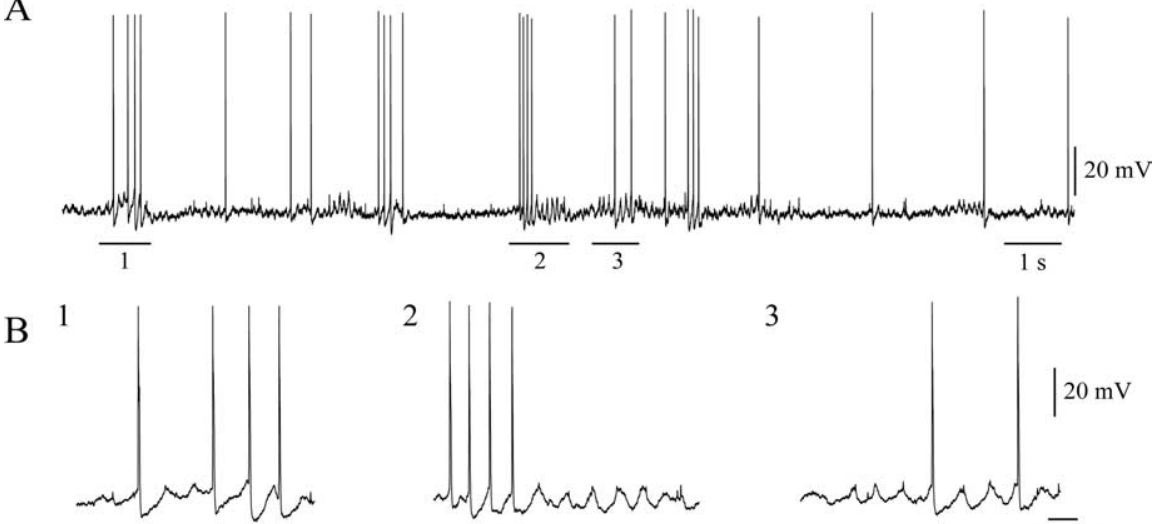

2

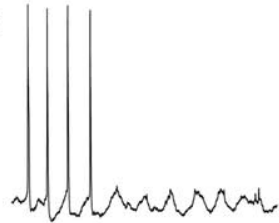

$\mathrm{C}$

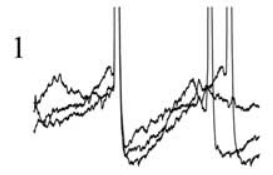

2

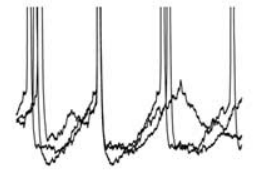

3

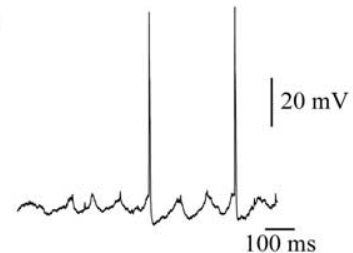

3
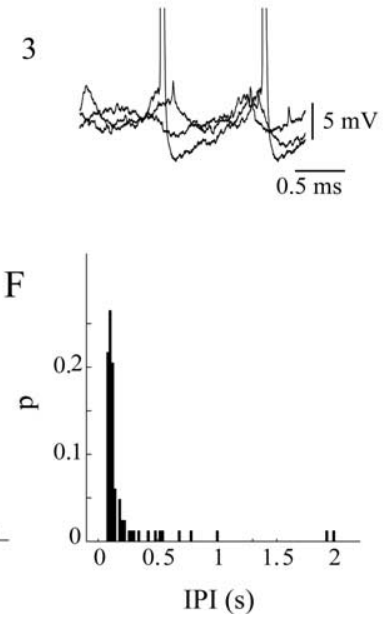

Figure 6. Subthreshold oscillatory activity characterizes the epochs of suprathreshold activity. $A, A$ typical example from a recording of an olivary neuron. $\boldsymbol{B}$, The three marked areas in $\boldsymbol{A}$ are shown at higher magnification. $\boldsymbol{C}$, Superposition of the spikes from each epoch reveals the close association between the subthreshold activity and the spikes. Note that in panel 3 , we superimposed two subthreshold events on the spike by aligning the peaks of subthreshold events with the deflection point of the spike. $\boldsymbol{D}$, Averaged autocorrelograms of all oscillatory epochs (after spike clipping) from this neuron reveals a $\sim 10 \mathrm{~Hz}$ rhythmicity. $\boldsymbol{E}$, The ISI histogram. $\boldsymbol{F}$, The IPI histogram. Note that both the ISI and IPI peak at an interval that corresponds to the $10 \mathrm{~Hz}$ rhythmicity.

likely to be the main source for the induction of these two rhythms. Furthermore, the independence of the distribution of IPIs on the presence of actions potential (Fig. 9) points to the same conclusion: that the oscillations are generated by network dynamics.

All of the spontaneous action potentials recorded from olivary neurons were of the high-threshold type. Although this finding is in line with previously published data from in vivo recording of olivary neurons (Crill and Kennedy, 1967), it contrasts with in vitro findings where low-threshold responses are frequently encountered. The differences in the firing modes between in vivo and in vitro conditions do not reflect altered intrinsic properties, because low-threshold spikes can be induced under in vivo conditions. Rather, these differences suggest that the level of network integrity modulate the mode of firing of the neurons. In in vivo conditions, cells are usually bombarded with inputs, resulting in significant depolarization of the dendrites. Thus, the threshold for eliciting a high-threshold action potential is quickly reached (Llinas and Yarom, 1981a). The prevalence of high-threshold spikes, which generate prolonged somatic depolarization, has significant functional implications. If depolarization lasts longer than the refractory period of the spike at the axon, it will evoke a high-frequency burst of action potentials that will propagate along the climbing fibers, all the way to the cerebellar cortex.
Indeed, the depolarizing wavelets that appear during the prolonged action potential are likely to reflect such axonal firing. Furthermore, a burst of climbing fiber triggered synapses at the cerebellar cortex has been reported previously (Eccles et al., 1966; Campbell and Hesslow, 1986).

The long-standing debate on the role of the climbing fiber inputs in cerebellar function is centered on the question of whether the inferior olive serves as a periodic clock for coordinating movements (Welsh and Llinas, 1993) in addition to its classical roles as a teaching signal (Marr, 1968; Albus, 1971; Ito, 1982; McCormick et al., 1985). The skepticism over the timing role of the inferior olive is attributable to the fact that only a few studies have reported rhythmicity of the olivary output (Welsh and Llinas, 1993; Sugihara et al., 1995; De Zeeuw et al., 1996; Lang et al., 1999; Lang, 2001, 2002; Blenkinsop and Lang, 2006). Many of the reports claim that these signals are random, and show no temporal structure (Keating and Thach, $1995,1997)$. In view of our observations obtained by intracellular recordings of olivary activity, we suggest that the absence of rhythmicity in CS activity is caused by a synergistic effect of the low firing rate of olivary neurons and the episodic nature of the rhythmic epochs. Independent epochs of subthreshold rhythmic activity will generate random spike trains provided that the overall firing rate is low. Thus, the absence of rhythmicity in the CS autocorrelograms does not imply that the output of olivary neurons is not rhythmic, but rather, that analyzing autocorrelograms of CS is insufficient to reveal the underlying rhythm. The only way to unravel this rhythm is to analyze the CS firing patterns at the population level (Welsh et al., 1995). In this way, the overall activity within a single epoch of rhythmic activity will undoubtedly reveal the underlying $\sim 10 \mathrm{~Hz}$ rhythm. It is noteworthy that previous in vivo intracellular recording from olivary neurons do not mention the existence of subthreshold oscillations (Crill and Kennedy, 1967; Crill, 1970; Ruigrok et al., 1990). The focus in these studies was on evoked responses, triggered by extracellular (Crill and Kennedy, 1967; Crill, 1970; Ruigrok et al., 1990) or intracellular stimulations (Crill and Kennedy, 1967; Crill, 1970). It could be that oscillations are diminished because of massive extracellular stimulation, caused by accumulation of neurotransmitters (Lang, 2001, 2002) or effects of stimulating serotonergic inputs known to block oscillations (Placantonakis et al., 2000). However, in the study by Ruigrok et al. (1990), in their Figure 2 B, it does seem that $6-8 \mathrm{~Hz}$ subthreshold oscillations are present even after stimulation.

Because the slow olivary rhythm described in this study apparently occurs solely in in vivo conditions, we hypothesize that it may reflect rhythmic inputs impinging onto the inferior olive nuclei. One such input can be serotonin, which was shown to effectively modulate the oscillatory properties of olivary neurons (Placantonakis et al., 2000). It is possible that the slow transitions 
between oscillatory and quiescent periods are the outcome of a pathological network state induced by the anesthesia. Nevertheless, these transitions clearly demonstrate the capacity of the network to shift olivary neurons from a quiescent to an oscillatory mode of operation. These transitions, that during wakeful conditions probably do not appear rhythmically, can serve as a gating mechanism. During the oscillatory mode, the input integrates with the intrinsic subthreshold activity and reaches the firing threshold. The suprathreshold activity can serve either as a timed error signal or as a timing signal per se. The latter support the observation that the output is locked to an underlying rhythmic process. This possibility implies that the underlying subthreshold rhythm is independent of the suprathreshold activity. Indeed, as shown in Figure 9, the period between waves of oscillatory activity is virtually independent of the presence of action potentials. Hence, action potentials do not seem to reset the rhythm. Because the rhythmicity of the cells is far from being highly periodic (Fig. 9), each cycle probably serves as an independent timing unit which is synchronized across many cells. Thus, the behavior of these cells suggests that the inferior olive has an important functional role in controlling events at short time intervals.

Previous studies have demonstrated that local application of either excitatory or inhibitory neurotransmitters to the inferior olive nuclei blocks CS rhythmicity (Lang, 2001, 2002). Furthermore, both excitatory and inhibitory synapses were shown to terminate at the sites of olivary gap-junctions (De Zeeuw et al., 1993), thus enabling them to shunt the current flowing between olivary neurons (Llinas et al., 1974). Hence, inputs arriving at the locations of olivary gap junctions could very well be responsible for the slow rhythm observed in olivary neurons. We postulate that such inputs, which can control the state of the olivary network, are likely to come from top-down sources such as the cerebellum or cerebrum. The deep cerebellar nuclei (DCN) could be one source of such top- down inputs that might shunt current flowing into gap junctions (De Zeeuw et al., 1993). Moreover, under similar experimental conditions, the membrane potential of PCs were shown to be bistable (Lowenstein et al., 2005). PCs in the up state inhibit DCN neurons. This inhibition will disinhibit the olivary network and drive it to oscillate (Lang, 2001, 2002). After transition of the PCs to the down state, DCN neurons are disinhibited, begin
A Distribution of frequencies of
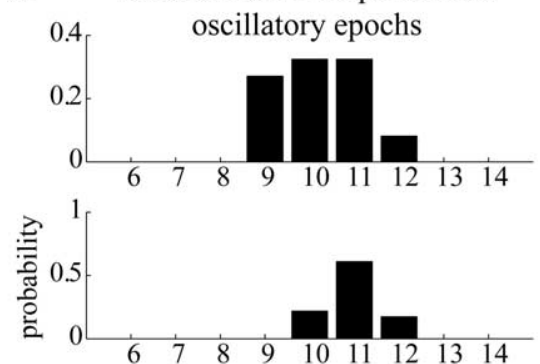

C
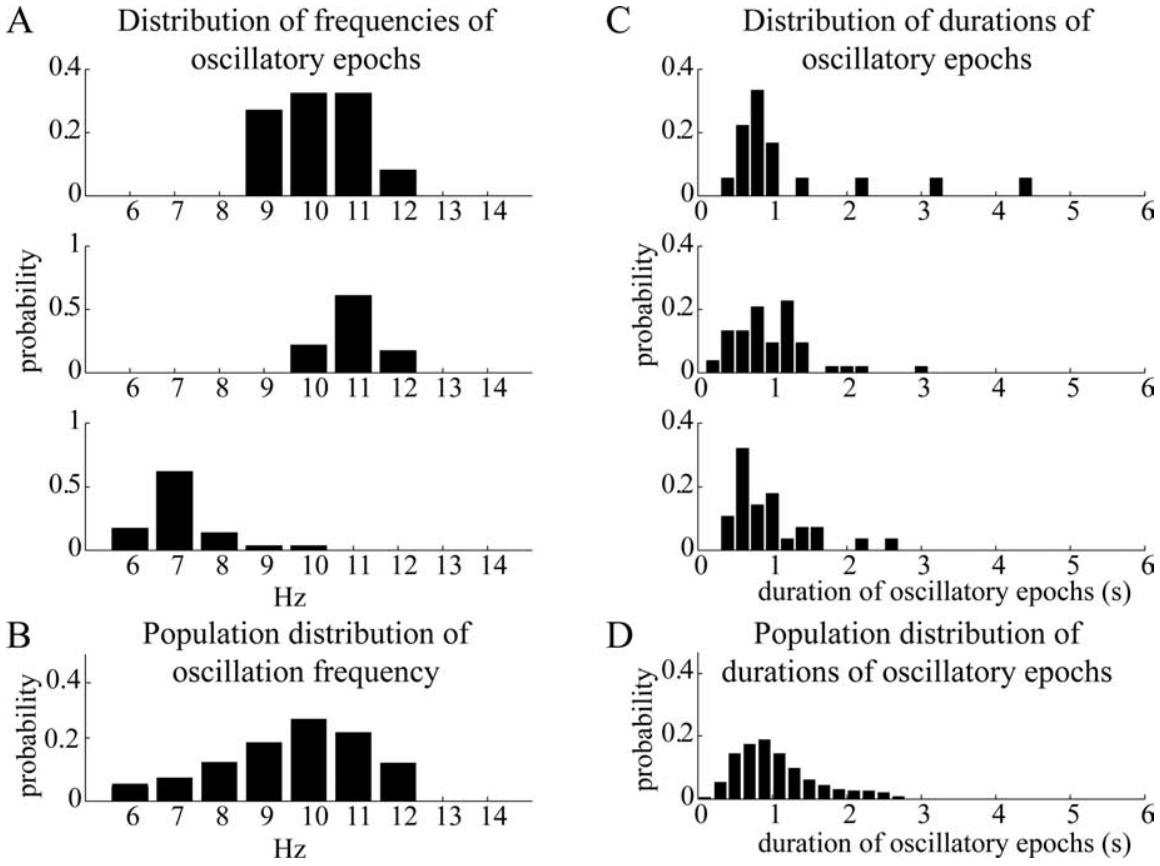

Figure 7. Distribution of duration of oscillatory epochs and their frequencies. For each neuron, the oscillatory epochs were identified and spectral analysis was performed to determine the duration of each epoch and its frequency of oscillations. $A$, The frequency distribution of all epochs from three different neurons. $\boldsymbol{B}$, The distribution of the average oscillation frequencies of 32 cells. Note that the distribution of the average frequency is wider than the distribution of frequencies in each neuron. $C$, The distribution of the durations of oscillatory epochs for three cells ( $200 \mathrm{~ms}$ bins). $\boldsymbol{D}$, The distribution of the average epoch durations. Note that population distribution is similar to the distribution of the duration of single neurons.
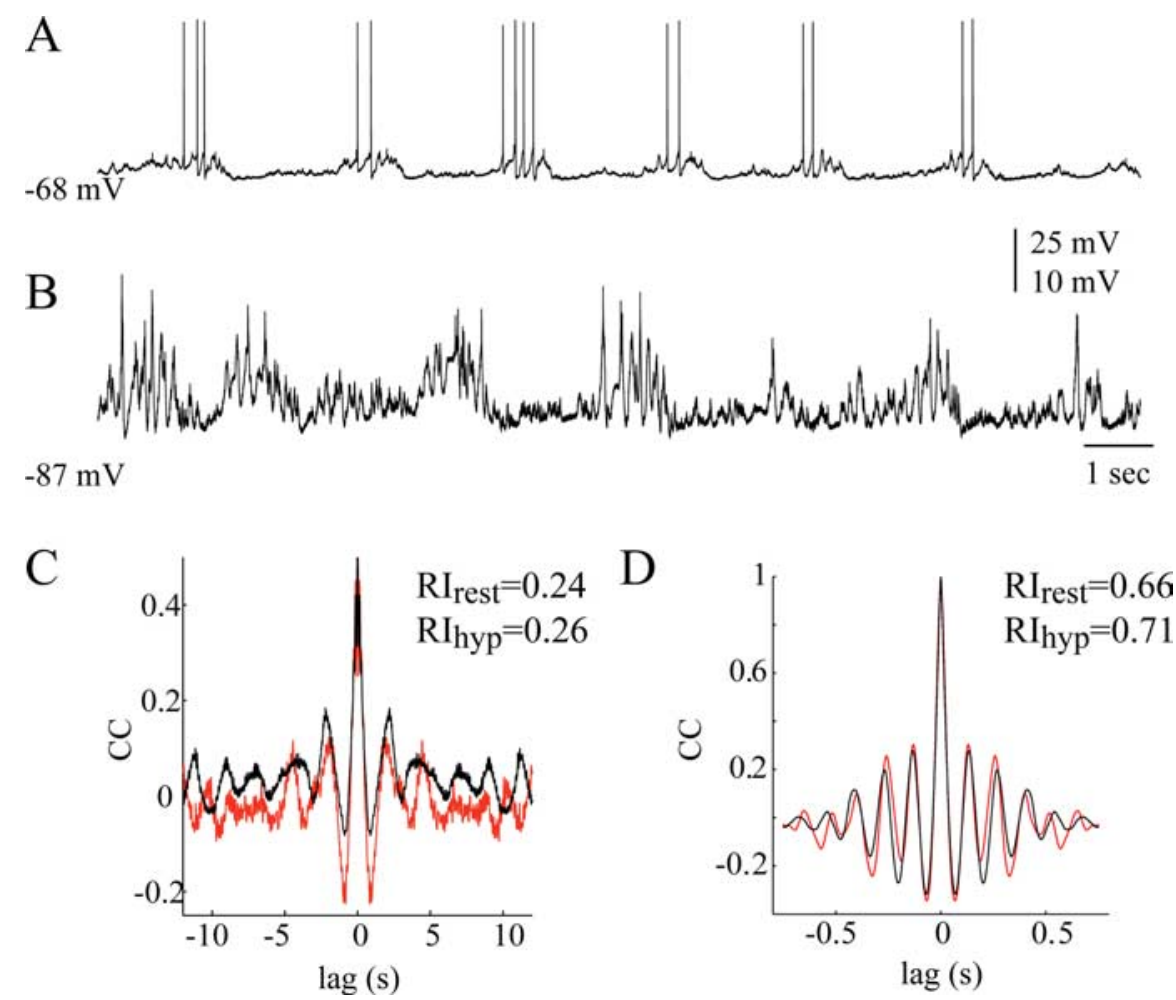

Figure 8. The slow and the fast rhythms are voltage independent. $A$, An example of a 10 -s-long recording in a resting condition. $\boldsymbol{B}$, An example of a 10-s-long recording in a hyperpolarized condition (direct current of $400 \mathrm{pA}$ was injected). $\boldsymbol{C}$, The autocorrelogram of the resting and hyperpolarized conditions in black and red, respectively. $\boldsymbol{D}$, The average autocorrelogram of the oscillatory epochs. Note that hyperpolarizing the membrane potential did not have prominent effects on the slow or fast rhythms. 




C

period $=90 \mathrm{~ms} ;$ spikes $=116$

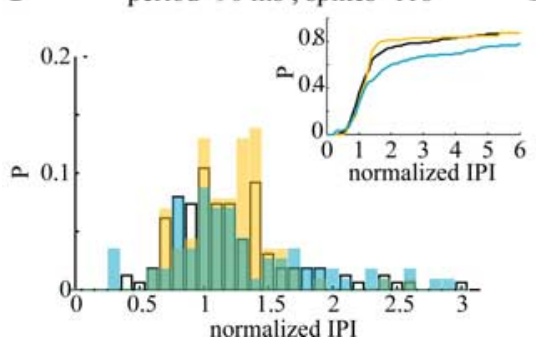

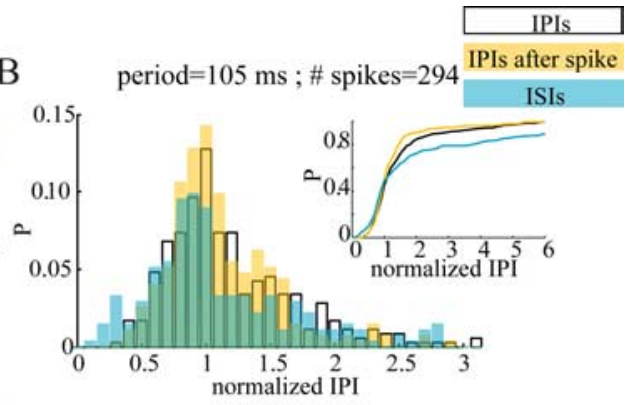

period=103 $\mathrm{ms}$; \# spikes=123

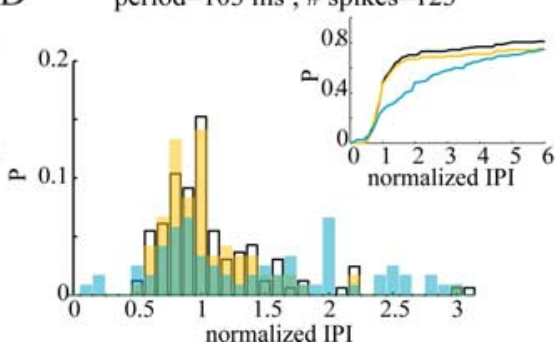

Figure 9. The distribution of the periods of cycles is not affected by the presence of action potential. The peaks of the subthreshold oscillations were detected and the IPI calculated. Each IPI was normalized by the most frequent IPI (i.e., cycle period). The population of IPIs was divided into two groups: the IPIs of peaks that did not follow action potentials (empty bars) and the IPIs of peaks that followed action potentials (yellow). Four examples of the distribution of the normalized IPIs are shown. In blue are the ISI histograms normalized to the same cycle period as the IPIs. The histograms show a similar distribution, indicating that spikes do not tend to induce changes in the durations of cycles following them. Note that overlap between the IPIs that follow spikes and the ISIs are colored green.

firing, and, thus, uncouple the olivary network. There is one prerequisite for this to work: the transition to and from the up state among PCs converging to the same DCN neurons needs to be synchronized. If indeed Purkinje cells control olivary oscillations via the DCN, it provides the system with the capacity to generate patterned activity that can then be used for temporal coordination of behavior.

\section{References}

Albus J (1971) A theory of cerebellar function. Math Biosci 10:25-61.

Andersson G, Armstrong D (1986) Complex spikes in Purkinje cells in the lateral vermis (b zone) of the cat cerebellum during locomotion. J Physiol (Lond) 385:107-134.

Blenkinsop TA, Lang EJ (2006) Block of inferior olive gap junctional coupling decreases Purkinje cell complex spike synchrony and rhythmicity. J Neurosci 26:1739-1748.

Campbell N, Hesslow G (1986) The secondary spikes of climbing fibre responses recorded from Purkinje cell axons in cat cerebellum. J Physiol (Lond) 377:225-235.

Chorev E, Manor Y, Yarom Y (2006) Density is destiny-on the relation between quantity of T-Type $\mathrm{Ca}^{2+}$ channels and neuronal electrical behavior. CNS Neurol Disord Drug Targets 5:655-662.

Crill WE (1970) Unitary multiple-spiked responses in cat inferior olive nucleus. J Neurophysiol 33:199-209.

Crill WE, Kennedy TT (1967) Inferior olive of the cat: intracellular recording. Science 157:716-718.

De Zeeuw C, Wentzel P, Mugnaini E (1993) Fine structure of the dorsal cap of the inferior olive and its GABAergic and non-GABAergic input from the nucleus prepositus hypoglossi in rat and rabbit. J Comp Neurol 327:63-82.

De Zeeuw CI, Lang EJ, Sugihara I, Ruigrok TJH, Eisenman LM, Mugnaini E, Llinas R (1996) Morphological correlates of bilateral synchrony in the rat cerebellar cortex. J Neurosci 16:3412-3426.

Devor A, Yarom Y (2002a) Generation and propagation of subthreshold waves in a network of inferior olivary neurons. J Neurophysiol 87:3059-3069.

Devor A, Yarom Y (2002b) Coherence of subthreshold activity in coupled inferior olivary neurons. Ann NY Acad Sci 978:508-.
Eccles JC, Llinas R, Sasaki K (1966) The excitatory synaptic action of climbing fibers on the Purkinje cells of the cerebellum. J Physiol (Lond) 182:268-296.

Friedberg MH, Lee SM, Ebner FF (1999) Modulation of receptive field properties of thalamic somatosensory neurons by the depth of anesthesia. J Neurophysiol 81:2243-2252.

Ito M (1982) Experimental verification of MarrAlbus' plasticity assumption for the cerebellum. Acta Biol Acad Sci Hung 33:189-199.

Keating JG, Thach WT (1995) Nonclock behavior of inferior olive neurons: interspike interval of Purkinje cell complex spike discharge in the awake behaving monkey is random. J Neurophysiol 73:1329-1340.

Keating JG, Thach WT (1997) No clock signal in the discharge of neurons in the deep cerebellar nuclei. J Neurophysiol 77:2232-2234.

Kim JH, Wang JJ, Ebner TJ (1987) Climbing fiber afferent modulation during treadmill locomotion in the cat. J Neurophysiol 57:787-802.

Kitazawa S, Wolpert DM (2005) Rhythmicity, randomness and synchrony in climbing fiber signals. Trends Neurosci 28:611-619.

Lampl I, Yarom Y (1993) Subthreshold oscillations of the membrane potential: a functional synchronizing and timing device. J Neurophysiol 70:2181-2186.

Lampl I, Yarom Y (1997) Subthreshold oscillations and resonant behavior: two manifestations of the same mechanism. Neuroscience 78:325-341.

Lang EJ (2001) Organization of olivocerebellar activity in the absence of excitatory glutamatergic input. J Neurosci 21:1663-1675.

Lang EJ (2002) GABAergic and glutamatergic modulation of spontaneous and motor-cortex-evoked complex spike activity. J Neurophysiol 87:1993-2008.

Lang EJ, Sugihara I, Welsh JP, Llinas R (1999) Patterns of spontaneous Purkinje cell complex spike activity in the awake rat. J Neurosci 19:2728-2739.

Leznik E, Makarenko V, Llinas R (2002) Electrotonically mediated oscillatory patterns in neuronal ensembles: an in vitro voltage-dependent dyeimaging study in the inferior olive. J Neurosci 22:2804-2815.

Llinas R, Yarom Y (1981a) Electrophysiology of mammalian inferior olivary neurones in vitro. Different types of voltage-dependent ionic conductances. J Physiol (Lond) 315:549-567.

Llinas R, Yarom Y (1981b) Properties and distribution of ionic conductances generating electroresponsiveness of mammalian inferior olivary neurones in vitro. J Physiol (Lond) 315:569-584.

Llinas R, Baker R, Sotelo C (1974) Electrotonic coupling between neurons in cat inferior olive. J Neurophysiol 37:560-571.

Llinas RR, Yarom Y (1986) Oscillatory properties of guinea-pig inferior olivary neurons and their pharmacological modulation: and in-vitro study. J Physiol (Lond) 376:163-182.

Lou JS, Bloedel JR (1986) The responses of simultaneously recorded Purkinje cells to the perturbations of the step cycle in the walking ferret: a study using a new analytical method-the real-time postsynaptic response (RTPR). Brain Res 365:340-344.

Lou JS, Bloedel JR (1988a) A new conditioning paradigm: conditioned limb movements in locomoting decerebrate ferrets. Neurosci Lett 84:185-190.

Lou JS, Bloedel JR (1988b) A study of cerebellar cortical involvement in motor learning using a new avoidance conditioning paradigm involving limb movement. Brain Res 445:171-174.

Lou JS, Bloedel JR (1992) Responses of sagittally aligned Purkinje cells during perturbed locomotion: synchronous activation of climbing fiber inputs. J Neurophysiol 68:570-580.

Lowenstein Y, Severine M, Chadderton P, Kazauo K, Sompolinsky H, Yarom Y, Hausser M (2005) Bistability of cerebellar Purkinje cells modulated by sensory stimulation. Nat Neurosci 8:202-211.

Makarenko V, Llinas R (1998) Experimentally determined chaotic phase 
synchronization in a neuronal system. Proc Natl Acad Sci USA 95:15747-15752.

Manor Y, Rinzel J, Segev I, Yarom Y (1997) Low-amplitude oscillations in the inferior olive: a model based on electrical coupling of neurons with heterogeneous channel densities. J Neurophysiol 77:2736-2752.

Marr D (1968) A theory of cerebellar cortex. J Physiol (Lond) 202:437-470.

McCormick DA, Steinmetz JE, Thompson RF (1985) Lesions of the inferior olivary complex cause extinction of the classically conditioned eyeblink response. Brain Res 359:120-130.

Placantonakis DG, Schwarz C, Welsh JP (2000) Serotonin suppresses subthreshold and suprathreshold oscillatory activity of rat inferior olivary neurones in vitro. J Physiol (Lond) 524:833-851.

Placantonakis DG, Bukovsky AA, Aicher SA, Kiem H-P, Welsh JP (2006) Continuous electrical oscillations emerge from a coupled network: a study of the inferior olive using lentiviral knockdown of Connexin36. J Neurosci 26:5008-5016.

Ruigrok T, De Zeeuw C, van Dijken H, voogd J (1990) Intracellular labeling of neurons in the medial accessory olive of the cat: I. Physiology and light microscopy. J Comp Neurol 300:462-477.

Schweighofer N, Doya K, Kawato M (1999) Electrophysiological properties of inferior olive neurons: a compartmental model. J Neurophysiol 82:804-817.
Sotelo C, Llinas R, Baker R (1974) Structural study of inferior olivary nucleus of the cat: morphological correlates of electrotonic coupling. J Neurophysiol 37:541-559.

Steinmetz J, Lavond D, Thompson R (1989) Classical conditioning in rabbits using pontine nucleus stimulation as a conditioned stimulus and inferior olive stimulation as an unconditioned stimulus. Synapse 3:225-233.

Sugihara I, Lang EJ, Llinas R (1995) Serotonin modulation of inferior olivary oscillations and synchronicity: a multiple-electrode study in the rat cerebellum. Eur J Neurosci 7:521-534.

Welsh JP, Llinas R (1993) On the cerebellum and motor learning. Curr Opin Neurobiol 3:958-965.

Welsh JP, Lang EJ, Suglhara I, Llinas R (1995) Dynamic organization of motor control within the olivocerebellar system. Nature 374:453-457.

Wentlandt K, Carlton S, Kushnir M, Naus CC, El-Beheiry H (2005) General anesthetics attenuate gap junction coupling in P19 cell line. J Neurosci Res 81:746-752.

Wentlandt K, Samoilova M, Carlen P, El-Beheiry H (2006) General anesthetics inhibit gap junction communication in cultured organotypic hippocampal slices. Anesth Analg 102:1989-1991.

Yarom Y, Cohen D (2002) The olivocerebellar system as a generator of temporal patterns. Ann NY Acad Sci 978:122-134. 